BULL. AUSTRAL. MATH. SOC.

VOL. 17 (1977), 419-421.

\title{
A note on injectors in finite soluble groups
}

\section{John Cossey}

Groups of nilpotent length four containing a subgroup which covers and avoids the same chief factors as an $\underline{\underline{X}}$-injector for some Fitting class $\underline{\underline{X}}$ but which is not itself an $\underline{\underline{X}}$-injector have been constructed by F.P. Lockett (in his $\mathrm{PhD}$ thesis) and T.R. Berger and John Cossey (in preparation). Graham A. Chambers (J. Algebra 16 (1970), 442-455) has shown that such a subgroup cannot exist in a group of $p$-length one for all primes $p$. The main result of this paper closes the small gap remaining: it includes Chambers' result and establishes also that such a subgroup cannot exist in a group of nilpotent length three.

Let $\underline{\underline{X}}$ be a Fitting class and $G$ a (finite soluble) group. Following Chambers [2], we shall say that the $\underline{=}$-injectors of $G$ are characterized by their cover-avoidance property if every subgroup of $G$ which covers and avoids the same chief factors as an X-injector is itself an $\underline{\underline{X}}$-injector. Chambers ([2], Theorem 4.3) has shown that if $G$ is a group of $p$-length one for all primes $p$, the $x$-injectors of $G$ are characterized by their cover-avoidance property. Lockett [3] and Berger and the author [1] have given examples of groups of nilpotent length four in which there are injectors not characterized by their cover-avoidance property.

The aim of this note is to fill the very small gap remaining by proving

Received 4 July 1977. 
THEOREM. Let $\underline{x}$ be a Fitting class of characteristic $\pi, G$ a group, and put $U(G) / 0_{\pi},(G)=\left(G / O_{\pi},(G)\right)_{\underline{X}}$. If $G / U(G)$ has p-length one for all $p \in \pi$, the $\mathrm{X}$-injectors of $G$ are characterized by their coveravoidance property.

Before proving the theorem, we note that Theorem 4.3 of Chambers [2] is an immediate corollary, and that if $G$ has nilpotent length three, $G / U(G)$ is metanilpotent and so of $p$-length one for all primes $p$, giving

COROLLARY. Let $\underline{x}$ be a Fitting class and $G$ a group of nilpotent length three. Then the $\mathrm{X}$-injectors of $G$ are characterized by their cover-avoidance property.

To prove the theorem, it will be enough, by Chambers [2], Theorem 2.6, to show that an $\mathrm{X}$-injector of $G$ is $p$-normally embedded for all primes $p$ (a subgroup $H$ of $G$ is said to be $p$-normally embedded for a prime $p$ if, when $P$ is a Sylow $p$-subgroup of $H, P$ is a sylow $p$-subgroup of its normal closure).

We proceed by induction. The theorem is clearly true for groups of order 1 , so suppose it is true for all groups $H$ with $H / U(H)$ of $p$-length one for all $p \in \pi$, and $|H|<|G|$, and suppose $G / U(G)$ has $p$-length one for all $p \in \pi$. If $X$ is an $X$-injector of $G$, then clearly $X$ is $p$-normally embedded for $p \in \pi^{\prime}$. Hence we need only consider $p \in \pi$.

Let $N$ be a maximal normal subgroup of $G$ : then $N / U(N)$ has

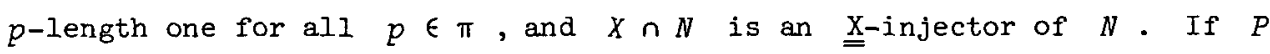
is a sylow $p$-subgroup of $X \cap N, P$ is a sylow $p$-subgroup of its normal closure in $N, M$ say. The X-injectors of $N$ form a characteristic conjugacy class, and hence so do the Sylow $p$-subgroups of injectors: it follows that $M$ is characteristic in $N$, and hence normal in $G$. If either $|G / N| \neq p$, or $X \subseteq N$, then $P$ is also a sylow $p$-subgroup of $X$, and $X$ is $p$-normally embedded in $G$.

Thus we may assume that every maximal normal subgroup has index $p$, and no maximal normal subgroup contains $X$. If $V / U(G)$ is the nilpotent residual of $G / U(G)$, then $G / V$ is a $p$-group, $V X=G$, and since $G / U(G)$ has $p$-length one, $V / U(G)$ is a $p^{\prime}$-group. It follows immediately that a Sylow $p$-subgroup of $X$ is also a sylow $p$-subgroup of $G$, and hence $X$ 
is $p$-normally embedded in $G$, completing the proof.

\section{References}

[1] T.R. Berger and John Cossey, "More Fitting formations", in preparation.

[2] Graham A. Chambers, "p-normally embedded subgroups of finite soluble groups", J. Algebra 16 (1970), 442-455.

[3] F.P. Lockett, "On the theory of Fitting classes of finite soluble groups" (PhD thesis, University of Warwick, Coventry, 1971).

Department of Pure Mathematics, Faculty of Arts,

Australian National University, Canberra, ACT. 